\title{
Molecular characterization of KU70 and KU80 homologues and exploitation of a KU70-deficient mutant for improving gene deletion frequency in Rhodosporidium toruloides
}

\author{
Chong Mei John Koh, Yanbin Liu, Moehninsi, Minge Du and Lianghui Ji*
}

\begin{abstract}
Background: Rhodosporidium toruloides is a $\beta$-carotenoid accumulating, oleaginous yeast that has great biotechnological potential. The lack of reliable and efficient genetic manipulation tools have been a major hurdle blocking its adoption as a biotechnology platform.

Results: We report for the first time the development of a highly efficient targeted gene deletion method in $R$. toruloides ATCC 10657 via Agrobacterium tumefaciens-mediated transformation. To further improve targeting frequency, the KU70 and KU80 homologs in $R$. toruloides were isolated and characterized in detail. A KU70-deficient mutant ( $\triangle \mathrm{ku} 70 \mathrm{e})$ generated with the hygromycin selection cassette removed by the Cre-loxP recombination system showed a dramatically improved targeted gene deletion frequency, with over $90 \%$ of the transformants being true knockouts when homology sequence length of at least $1 \mathrm{~kb}$ was used. Successful gene targeting could be made with homologous flanking sequences as short as $100 \mathrm{bp}$ in the $\triangle \mathrm{ku} 70 \mathrm{e}$ strain. KU70 deficiency did not perturb cell growth although an elevated sensitivity to DNA mutagenic agents was observed. Compared to the other well-known oleaginous yeast, Yarrowia lipolytica, R. toruloides KU70/KU80 genes contain much higher density of introns and are the most GC-rich KU70/KU80 genes reported.

Conclusions: The KU70-deficient mutant generated herein was effective in improving gene deletion frequency and allowed shorter homology sequences to be used for gene targeting. It retained the key oleaginous and fast growing features of $R$. toruloides. The strain should facilitate both fundamental and applied studies in this important yeast, with the approaches taken here likely to be applicable in other species in subphylum Pucciniomycotina.
\end{abstract}

Keywords: Nonhomologous end-joining (NHEJ), Rhodosporidium toruloides, Oleaginous yeast, Agrobacterium tumefaciens-mediated transformation (ATMT), Homologous recombination

\section{Background}

Rhodosporidium toruloides is a $\beta$-carotenoid accumulating oleaginous yeast in subphylum Pucciniomycotina [1]. Able to accumulate more than $70 \%$ of its dry cell mass as triacylgleride with similar chemical composition to those of plants from ultra-high density fermentation [2-4], $R$. toruloides is regarded as a great host with vast biotechnological potential to produce single cell oil, which may find wide spread applications in staple food,

\footnotetext{
* Correspondence: jilh@tll.org.sg

Biomaterials and Biocatalysts Group, Temasek Life Sciences Laboratory, 1

Research Link, National University of Singapore, Singapore 117604, Singapore
}

animal feed, biodiesel, surfactant and raw material for industrial polymers [3,5]. Although studies have been done to optimize lipid yield through high-density fermentation [2], there are scarce reports on the rational genetic engineering to improve lipid accumulation or fatty acid profiles in $R$. toruloides. To date, there are no reverse genetic studies reported in $R$. toruloides. With the advent of efficient and stable transformation method established using Agrobacterium tumefaciens-mediated transformation (ATMT) in $R$. toruloides [6], reverse genetic studies should become a real possibility. 
Targeted gene deletion, often referred as targeted gene knockout, is an essential tool for genetic engineering and reverse genetics. This is an important cornerstone to make any strains commercially competitive [7]. While targeted gene integration in model microorganisms, such as Saccharomyces cerevisiae and Schizosaccharomyces pombe, can be done with ease and high efficiency [8,9], it is a major obstacle in many industrially important species such as $R$. toruloides.

It has been proposed that DNA repair of doublestranded breaks by homologous recombination (HR) and non-homologous end-joining (NHEJ) operate competitively [10], and the predominance of NHEJ over HR has been regarded as the main cause of low gene targeting efficiency in fungi $[11,12]$. Correspondingly, one strategy to deal with low gene targeting efficiency in fungi is to improve the HR pathway $[11,13]$. The other strategy is to inhibit or eliminate the NHEJ pathway, thereby forcing the transformed DNA to be integrated via HR. With this approach, the frequency of HR has been found to be significantly improved with many reports of success in recent years through the disruption of NHEJ pathway by deleting one or more of its key components [12]. In eukaryotes, the main component of the NHEJ system is the DNA-dependent protein kinase (DNA-PK), a three-protein complex consisting of the DNA-dependent protein kinase catalytic subunit (DNA-PKcs) and the regulatory DNA-binding subunits, the Ku70/80 heterodimer [14]. The $\mathrm{Ku}$ heterodimer is an abundant nonspecific DNA-binding protein comprising of two tightly-associated subunits of about 70 and $83 \mathrm{kDa}$, named Ku70 and Ku80 respectively [15]. Both proteins exist in organisms ranging from fungi to human, and are arguably the defining proteins of NHEJ because of their sequence conservation [16].

Here, we report the isolation and characterization of KU70 and KU80 homologs in $R$. toruloides and the evaluation of a KU70-deficient mutant strain generated for improving gene deletion efficiency in $R$. toruloides.

\section{Results}

Isolation and characterization of Ku70 and Ku80 encoding genes in $R$. toruloides

Putative genes encoding the Ku70 and Ku80 homologues in the Rhodotorula glutinis ATCC 204091 (now re-named as Rhodosporidium toruloides ATCC 204091) genome were identified by tBLASTn search against the $R$. glutinis ATCC 204091 genome database at NCBI using the Ustilago maydis $\mathrm{Ku} 70$ and Ku80 sequences as the query (GenBank acc. no. XP_761295 and XP_761903 respectively). 5' and 3' RACEs were performed to obtain the full-length cDNA sequences. The KU70 cDNA contains a 2,118-nt open reading frame (ORF) flanked by 57-nt and 99-nt $5^{\prime}$ and $3^{\prime}$ untranslated region (UTR) respectively, while the KU80 cDNA contains a 2,766-nt ORF with 76-nt 5' UTR and 83-nt 3' UTR. Comparison of the cDNAs with the genomic sequences revealed that the KU70 mRNA spans over 3,047 bp containing 16 exons separated by 15 introns, whereas the KU80 mRNA spans over 3,426 bp containing 11 exons separated by 10 introns (Figure 1 ). All intronic sequences conformed strictly to the GT-AG rule [17], with a GC content of approximately $61 \%$, which is not significantly different to that of exonic sequences (Table 1). Sequencing of the 3,047 bp KU70 genomic region in $R$. toruloides ATCC 10657 revealed 100\% identity to that of $R$. toruloides ATCC 204091. A comparison with a number of other fungal homologues are shown in Table 1, which shows that $R$. toruloides KU70 and KU80 genes have the highest GC content and highest density of introns (1 in 196 nt on average).

The Ku70 ORF sequence was predicted to encode for a protein of 706 amino acids with a molecular weight of $79.5 \mathrm{kDa}$. Ku70 showed $25 \%$ to $30 \%$ identities to those from Homo sapiens, Neurospora crassa, Aspergillus niger and Cryptococcus neoformans, with the N. crassa Ku70 being the closest homologue (Figure 2). Analysis of Ku70 against the SUPERFAMILY database [23] revealed a Ku70 core domain (aa 288-589) that is flanked by a N-terminal "von Willebrand" A (vWA)-like domain (aa 31-54, 82258), and a C-terminal SAP domain (aa 631-663). The high sequence similarity and presence of signature domains conserved among Ku70 homologues suggest that the characterized Ku70 would be the key component of the NHEJ pathway in $R$. toruloides.

\section{Targeted gene deletion in wild type $R$. toruloides and generation of KU70 null mutants}

To see whether targeted gene deletion could be achieved in wild type $R$. toruloides, KU70 was used as the first deletion target. A derivative of $R$. toruloides ATCC 10657 (Rt1CE6, named WT hereafter, our unpublished data), which contained a $17 \beta$-estradiol inducible Cre recombinase gene stably integrated into the genome and allowed the recycling of hygromycin selection marker, was used in ATMT using the KU70 deletion construct, pKOKU70 (Figure 3A). Eight candidates out of 96 transformants were screened for loss of the targeted deletion region as judged by multiplex PCR (absence of KU7O PCR product and presence of GPD1 reference PCR product, data not shown). Further investigation using Southern blot analysis demonstrated that 5 out of 8 candidates were true KU70 deletion mutants without ectopic integration (Figure 3B). The mutant in lane 2 was therefore named $\Delta \mathrm{ku} 70$.

\section{Gene deletion frequency was improved in the $\Delta \mathrm{ku} 70$ mutant}

While the deletion of $K U 70$ was obtained with a relatively high frequency (5.2\%), deletion of the mating-type 


\section{A KU70

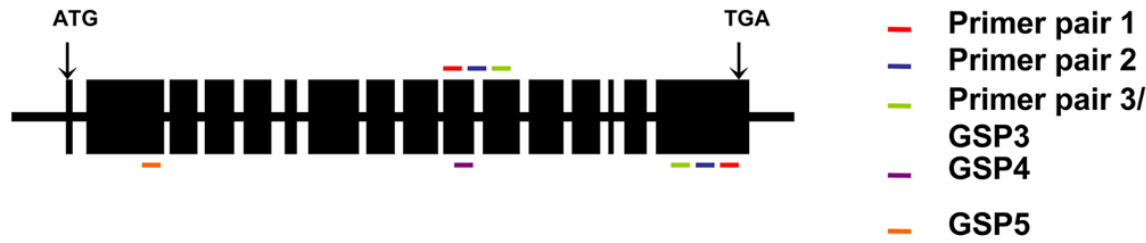

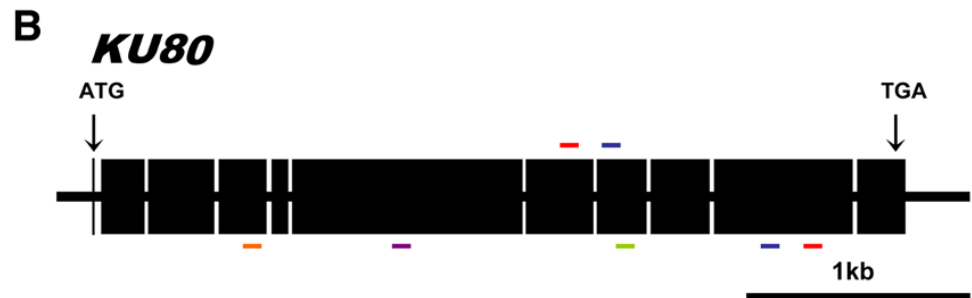

Figure 1 Genomic organization of KU70/80 from R. toruloides. (A) Genomic organization of KU70. (B) Genomic organization of KU80. Exons (indicated by black boxes) were identified by comparing the CDNAs and their corresponding genomic DNA sequences. The positions of translation initiation (ATG) and termination (TGA) codons are indicated by arrows. Colored bars indicate positions of gene-specific primers (GSPs) designed for RACEs, with those for 3' RACE shown on top and those for 5' RACE shown at the bottom.

specific gene STE20 and orotidine 5-phosphate decarboxylase gene URA3 [24,25] proved to be very difficult (Table 2). The low deletion frequency of STE2O and URA3 highlighted a need for an improved gene deletion system. To investigate if the $\Delta \mathrm{ku} 70$ strain generated earlier could be utilized for this purpose, the hygromycin selection cassette $\left(\mathrm{P}_{\text {GPDI }}:\right.$ hpt-3:: $\left.\mathrm{T}_{\text {nos }}\right)$ was excised to generate a marker-free $R$. toruloides KU70-deficient derivative $(\Delta \mathrm{ku} 70 \mathrm{e})$ by activating the Cre recombinase using human hormone 17 $\beta$-estradiol (Liu et al., unpublished data). As we found that high percentage of 5fluoroorotic acid (5-FOA) resistant transformants were not true deletion mutants of URA3 previouly, we decided to evaluate the deletion of CAR2 homologue as a fast assay for gene deletion frequency because it encodes a bifunctional protein catalyzing phytoene synthase and carotene cyclase that is essential in the biosynthesis of $\beta$-carotene $[25,26]$.

Using $U$. maydis Car2 [26] as a query for tBLASTn search against the $R$. toruloides ATCC 204091 genome database, a DNA fragment sharing high sequence homology to the query (GenBank acc. no. AVER02000018 from 396838 to 399094-nt, E-value $=1 \mathrm{E}-23$ ) was identified. CAR2 was successfully amplified using DNA template of $R$. toruloides ATCC 10657 using oligos Rt079 and Rt080. As expected, albino transformants was observed when WT was transformed with the CAR2 knockout construct pKOCAR2 (Figure 4A), and the color phenotype of transformants were stable after several rounds of subcultures (data not shown). Multiplex PCR and Southern blot

Table 1 Comparison of $K U 70 / 80$ organization between fungal homologues

\begin{tabular}{|c|c|c|c|c|c|c|c|c|}
\hline Gene & Strain & GenBank accession no. & CDS (nt) & CDS CG (\%) & Intron no. & Intron CG (\%) & Average intron length (nt) & Reference \\
\hline \multirow[t]{6}{*}{ KU70 } & N. crassa & AB177394 & 2046 & 51.4 & 2 & 45.4 & 54 & [18] \\
\hline & A. niger & EF061656 & 2283 & 50.7 & 5 & 45.4 & 67 & [19] \\
\hline & C. neoformans & XM_573016 & 1683 & 48.0 & 10 & 46.6 & 117 & [20] \\
\hline & Y. lipolytica & CR382129 & 1758 & 48.9 & 0 & - & - & {$[21,22]$} \\
\hline & & XM_501610 & & & & & & \\
\hline & R. toruloides & KF850470 & 2121 & 59.8 & 15 & 61.1 & 61 & This study \\
\hline \multirow[t]{5}{*}{ KU80 } & N. crassa & AB177395 & 2764 & 51.2 & 7 & 48.3 & 111 & [18] \\
\hline & C. neoformans & XM_568810 & 2511 & 47.9 & 13 & 43.4 & 53 & [20] \\
\hline & Y. lipolytica & CR382131 & 2181 & 48.6 & 1 & 37.5 & 48 & {$[21,22]$} \\
\hline & & XM_503443 & & & & & & \\
\hline & R. toruloides & KF850471 & 2769 & 62.1 & 10 & 61.1 & 66 & This study \\
\hline
\end{tabular}




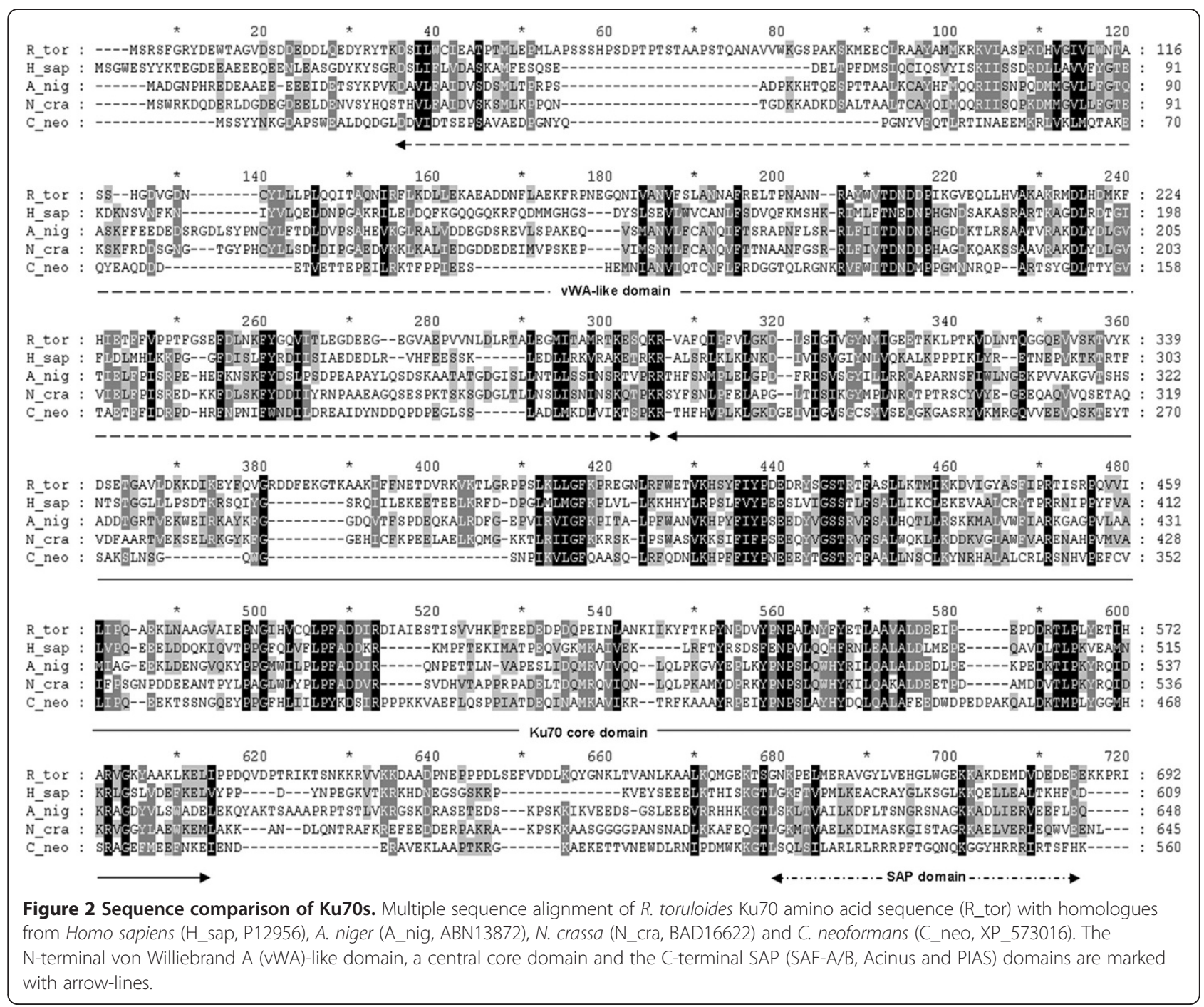

analysis further confirmed that all albino transformants tested were true car2 null mutants (Figure 4B). The albino phenotype was directly caused by the deletion of CAR2 because the phenotype was completely restored when reintegrating a wild type gene fragment (Additional file 1). Whereas the targeted deletion frequency for CAR2 was estimated to be $10.5 \%$ in WT, it was increased to $75.3 \%$ in the $\Delta$ ku70e background, a more than 7 -fold improvement. Dramatically increased gene deletion frequencies were also observed at both STE2O and URA3 loci (Table 2), with the deletions verified by Southern blot and phenotypic analyses (Figure 5).

\section{Effect of homology sequence length on deletion frequency}

To understand the effects of homology sequence length on gene deletion frequency, pKOCAR2 was modified to have various lengths of homology sequence, ranging from 50 to 1500 bp (Additional file 2). The minimum homology length necessary for CAR2 deletion in WT was at least $250 \mathrm{bp}$ with a gene deletion frequency of $0.7 \%$, while only $100 \mathrm{bp}$ was sufficient in the $\Delta \mathrm{ku} 70 \mathrm{e}$ strain, which gave gene deletion frequency of approximately $20 \%$. Homology length of at least $1 \mathrm{~kb}$ was required to achieve gene deletion frequency of more than $90 \%$ using the $\Delta$ ku70e strain (Table 3 ).

\section{Sensitivity of KU70 deficient mutant to DNA damaging agents}

Deficiency in $\mathrm{Ku}$ complex encoding genes have been linked to elevated sensitivity to DNA-damaging agents due to the defects in DNA repair [12]. As expected, the $\Delta$ ku70 strain displayed higher susceptibility to DNA damage induced by methyl methane sulfonate (MMS) and exposure to ultraviolet (UV) radiation compared to WT. The growth of both strains was repressed 


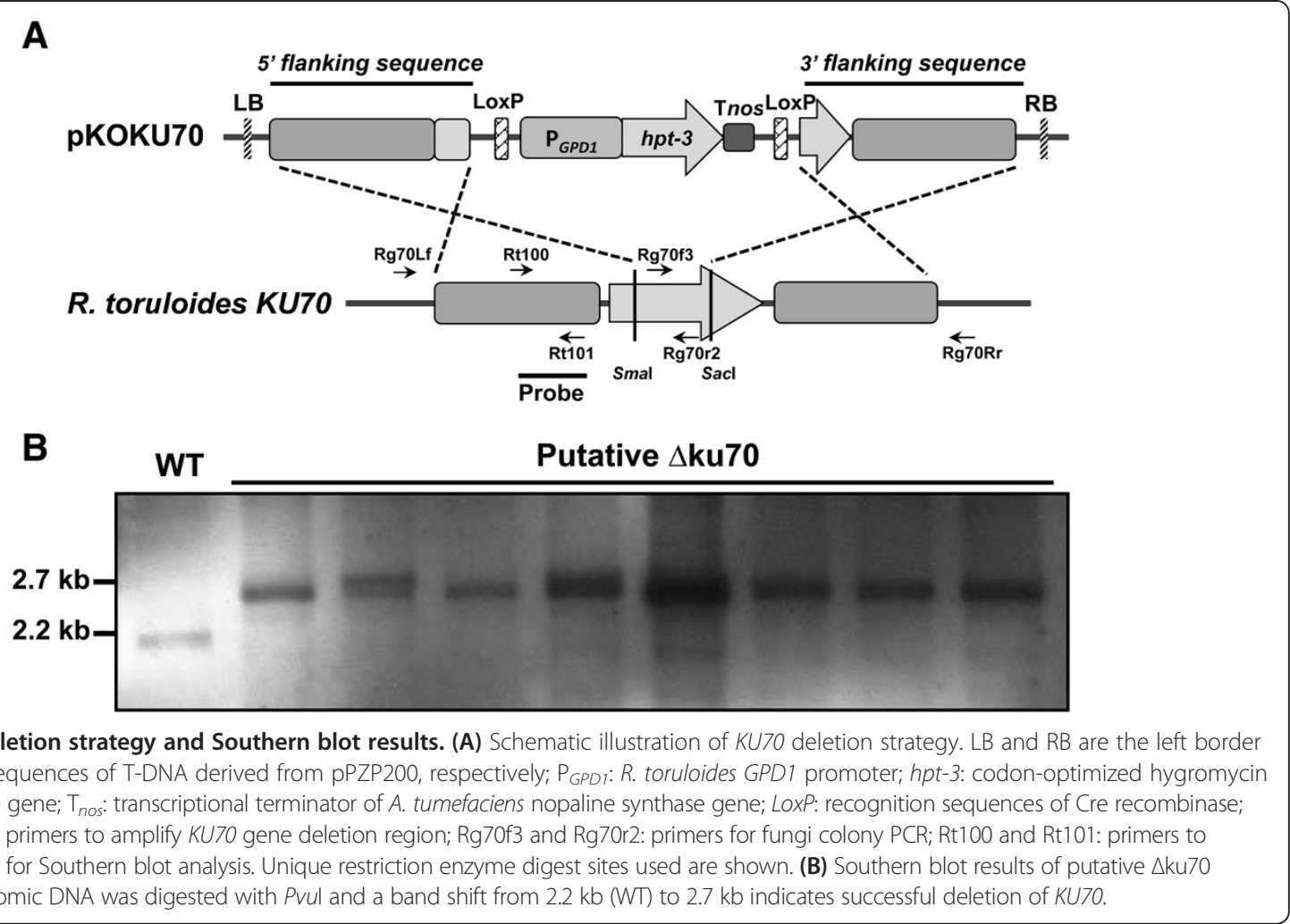

when MMS concentration and UV radiation reached $0.01 \%$ and $200 \mathrm{~J} / \mathrm{m}^{2}$ respectively (Figure 6). However, the KU70-deficient strain showed no obvious growth defects under normal growth conditions and its cell morphology was indistinguishable from WT. In addition, there were no significant differences in sugar consumption rate and fatty acid profile between WT and $\Delta \mathrm{ku} 70$ (Additional file 3).

\section{Discussion}

With more than $60 \%$ GC content, the KU70 and KU80 characterized here present the most GC-rich genes in the NHEJ-pathway reported so far. In terms of gene structure, both genes contain much higher density of introns than those of $Y$. lipolytica (Table 1), which is the best-studied oleaginous yeast to date. Not surprisingly,

\section{Table 2 Gene deletion frequency in WT and $\Delta$ ku70e} strains

\begin{tabular}{lccr}
\hline $\begin{array}{c}\text { Gene } \\
\text { target }\end{array}$ & $\begin{array}{c}\text { Homolgy } \\
\text { length }^{\mathbf{a}} \text { (bp) }\end{array}$ & \multicolumn{2}{c}{ Gene deletion frequency } \\
\cline { 3 - 4 } & 800 & $\mathbf{W T}$ & $\mathbf{\Delta}$ ku70e \\
\hline STE20 & 1000 & $0(560)$ & $2.1 \%(48)$ \\
URA3 & 750 & $0(48)$ & $95.8 \%(48)$ \\
CAR2 & $10.5 \%(6152)$ & $75.3 \%(885)$ \\
\hline
\end{tabular}

Note: ${ }^{a}$ Homology sequence length on each side of the hygromycin selection cassette; ${ }^{b}$ Number in parenthesis indicate number of transformants screened. homologues of $C$. neoformans, which is under the same Basidiomycota phylum, also have high density of introns (Table 1).

DSB repair can differ in heterochromatic and euchromatic regions of the genome and histone modifying factors play an important role in this process [28,29]. Recombination frequencies are known to vary in different genes even when assayed with the same technique and in the same genetic background [30]. Impairment of the NHEJ-pathway has proved to be effective in improving homologous recombination frequency in many eukaryotic hosts. However, the magnitude of improvement appears to vary considerably in different reports. With a homology sequence of approximately $750 \mathrm{bp}$, the CAR2 deletion frequency was improved 7.2-fold, from $10.5 \%$, in WT to $75.3 \%$ in the KU70-deficient mutant in $R$. toruloides. This is similar to the deletion of TRP1 in $Y$. lipolytica although substantially higher knockout frequencies have been reported for several genes in other fungi, for example, $N$. crassa, A. niger and $C$. neoformans (Additional file 4). Nevertheless, the $R$. toruloides STE2O gene remained very difficult to knockout even with the $\Delta$ ku70e mutant (Table 2). This demonstrates a positional effect and implies additional factors that regulate gene deletion in $R$. toruloides. As the STE2O gene is located between the mating type loci $R H A 2$ and $R H A 3$ in $R$. toruloides [24], it is possible that the gene is within a 


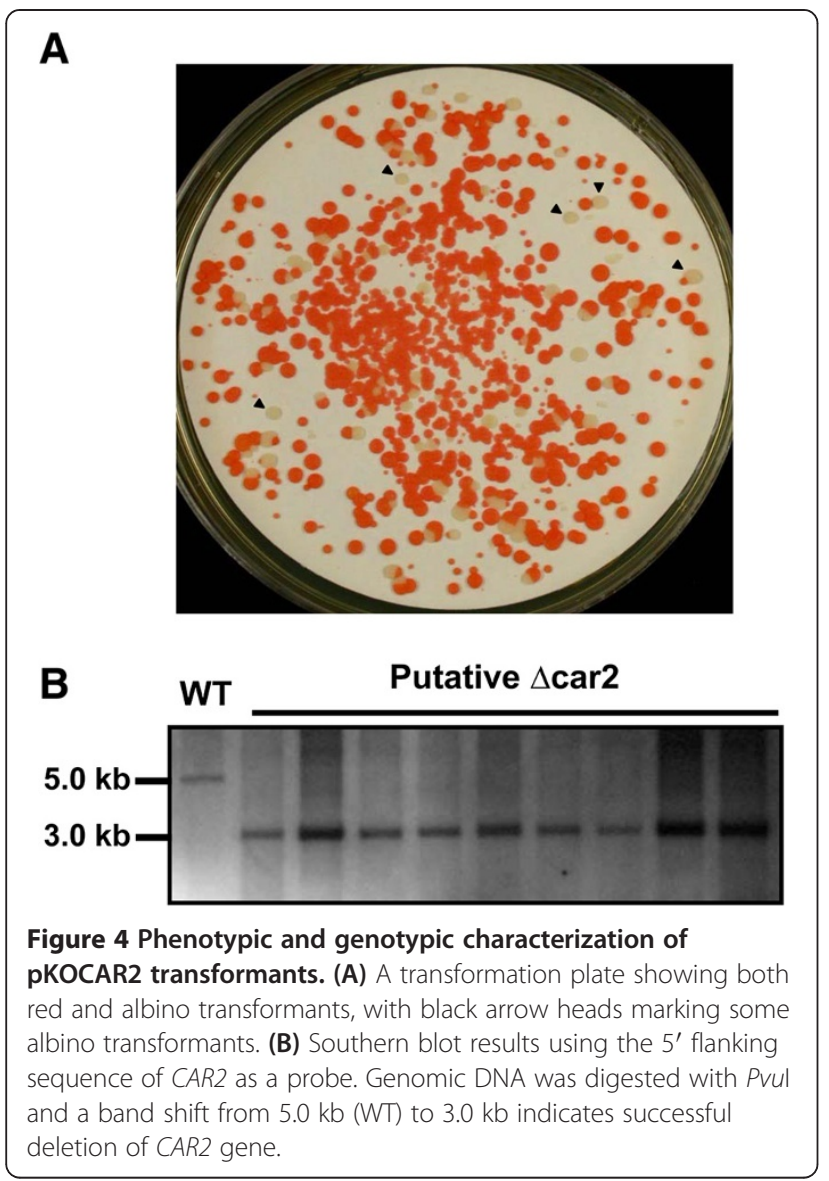

transcriptionally silenced chromatin as was reported for the mating type genes in a number of other fungi [31,32]. The low deletion frequency of STE2O suggests a potential role of chromatin structure and/or gene expression level in regulating DNA recombination in $R$. toruloides.

One of the drawbacks of NHEJ-deficient strains is its elevated sensitivity to DNA damage and the possibility of generating unwanted mutations [12]. Indeed, the KU70-deficient strain studied here showed increased sensitivity to MMS and UV radiation. However, the mutant did not show severe growth defects under normal growth conditions. With comparable sugar consumption rate and fatty acid profile to the WT, the $\Delta \mathrm{ku} 70$ and $\Delta$ ku70e strains should maintain much of the appeal of $R$. toruloides in industrial applications.

\section{Conclusions}

The KU70-deficient mutant generated herein was found to be effective in improving gene deletion frequency and retained the key oleaginous and fast growing features of $R$. toruloides. The strain should facilitate both fundamental and applied studies in this important yeast, with the approaches taken here likely to be applicable in other species in subphylum Pucciniomycotina.

\section{Methods}

Strains, media, and culture conditions

R. toruloides strain ATCC 10657 and ATCC 204091 (previously named Rhodotorula glutinis) were purchased from

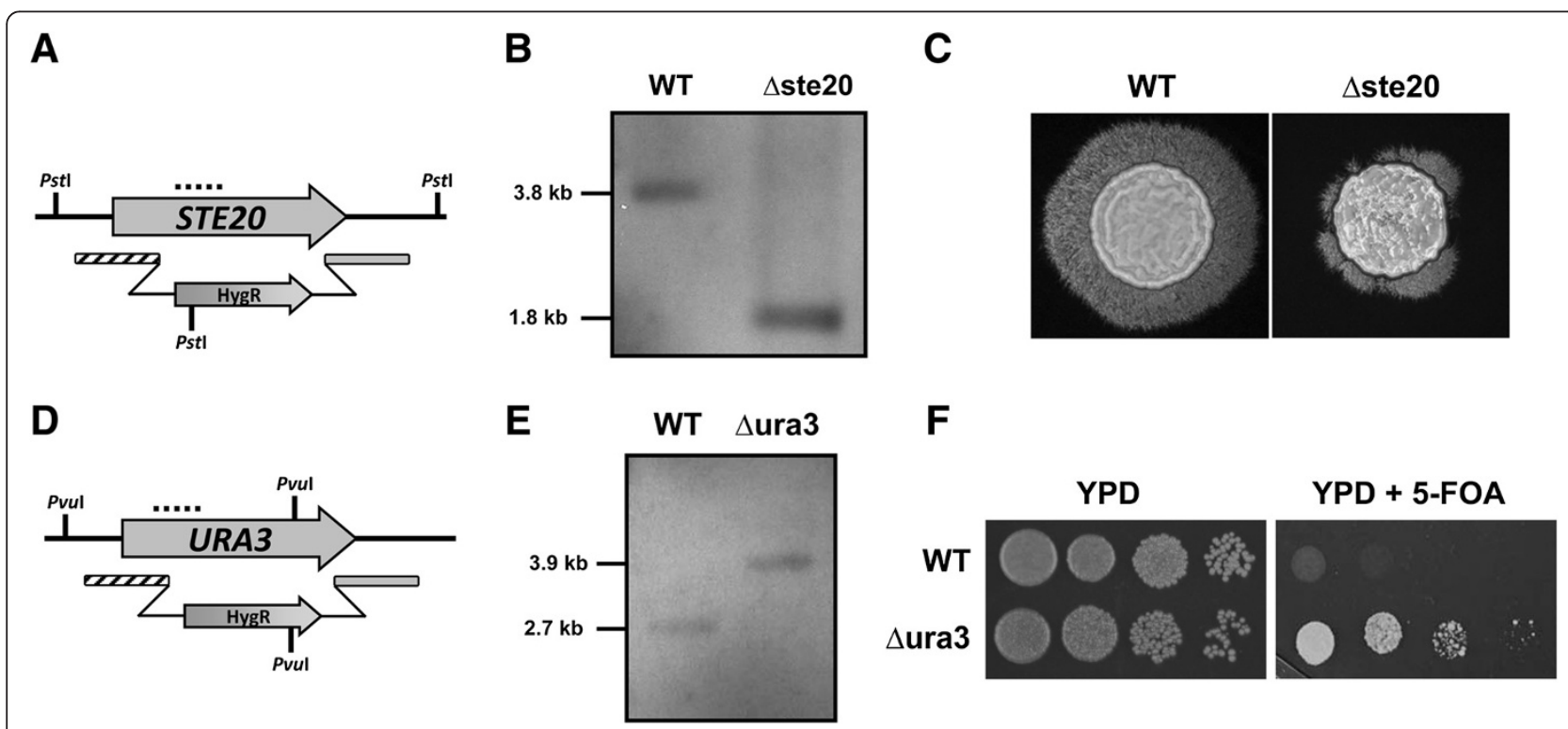

Figure 5 Targeted deletion of STE2O and URA3 genes. (A) and (D) Illustration of gene deletion constructs; (B) and (E): Southern blots using probes shown in (A) and (D); (C) Colony phenotype of WT and $\triangle$ ste20 strains mated with R. toruloides ATCC 10788; (F) Growth phenotype of WT and $\triangle$ ura3 strains derived from 10 -fold serially diluted cells. The latter showed resistance to $5-F O A(1 \mathrm{~g} / \mathrm{L})-$ a substrate that can be converted to a toxic intermediate by the URA3-encoded enzyme [27]. 
Table 3 Effects of homologous sequence length on CAR2 deletion frequency

\begin{tabular}{|c|c|c|c|}
\hline \multirow{2}{*}{$\begin{array}{l}\text { Homology } \\
\text { length } \\
\text { (bp) }\end{array}$} & \multicolumn{2}{|c|}{ Gene deletion frequency $^{a}$} & \multirow{2}{*}{$\begin{array}{l}\text { Improvement } \\
\text { (folds) }\end{array}$} \\
\hline & WT & $\Delta \mathrm{ku} 70 \mathrm{e}$ & \\
\hline 50 & $0(780)$ & $0(8)$ & - \\
\hline 100 & $0(620)$ & $21.4 \%(14)$ & - \\
\hline 250 & $0.7 \%(1668)$ & $30.3 \%(33)$ & 43.3 \\
\hline 500 & $11.2 \%(2124)$ & $67.0 \%(778)$ & 6 \\
\hline 750 & $10.5 \%(6152)$ & $75.3 \%(885)$ & 7.2 \\
\hline 1000 & $30.4 \%(2280)$ & $91.7 \%(2196)$ & 3 \\
\hline 1500 & $20.5 \%(2730)$ & $91.0 \%$ (4304) & 4.4 \\
\hline
\end{tabular}

Note: ${ }^{a}$ Number in parenthesis indicate number of transformants screened.

American Type Culture Collection (ATCC, Manassas, VA, USA) and cultured at $28^{\circ} \mathrm{C}$ in YPD broth (1\% yeast extract, $2 \%$ peptone, $2 \%$ glucose, w/v) or on potatodextrose agar (PDA). A. tumefaciens strain AGL1 [33] was grown at $28^{\circ} \mathrm{C}$ in either liquid or solid $2 \mathrm{YT}$ medium (1.6\% tryptone, $1 \%$ yeast extract, $0.5 \% \mathrm{NaCl}, \mathrm{pH} 7.5)$. Escherichia coli XL1-Blue was cultured at $37^{\circ} \mathrm{C}$ in LuriaBertani (LB) broth or on LB agar for routine recombinant DNA work.

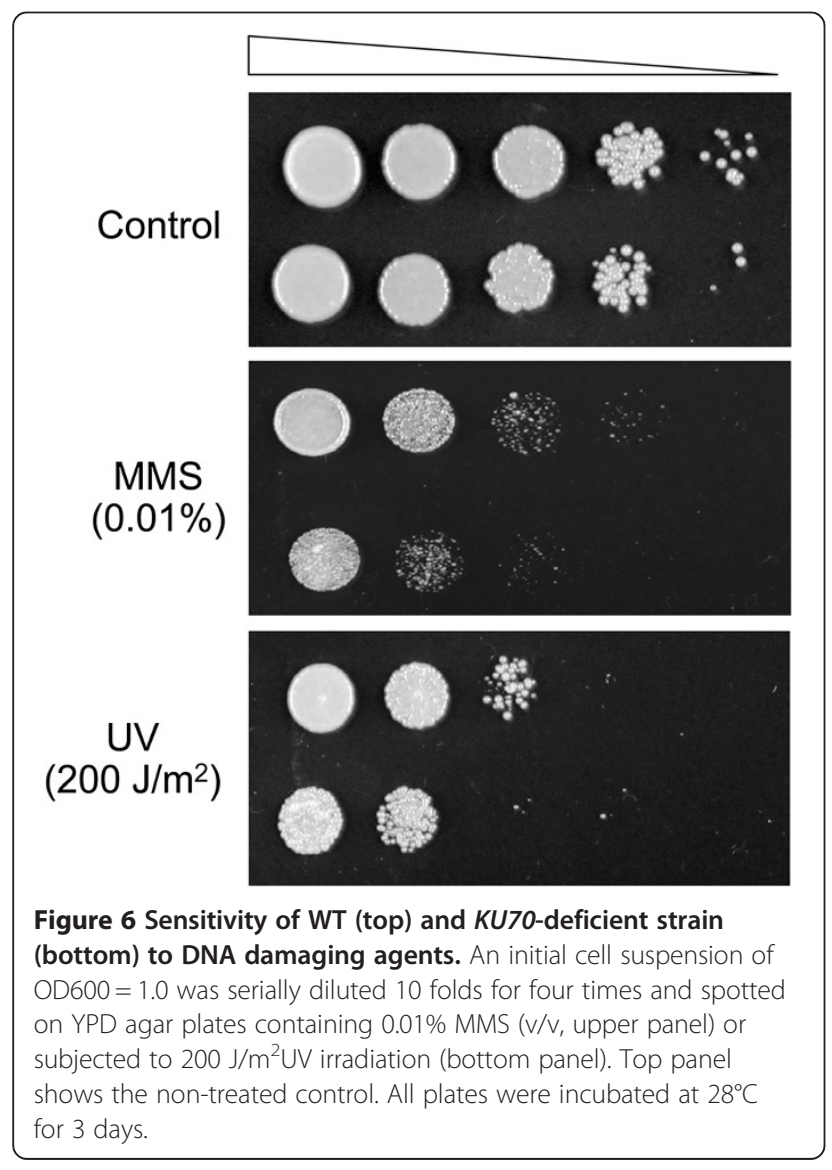

Rapid amplification of cDNA ends (RACE)

The SMARTer $^{\mathrm{TM}}$ RACE cDNA Amplification Kit (Clontech, Mountain CA, USA) was used to determine the full-length sequences of KU70 and KU80 RNA transcripts according to the manufacturer's instruction. For KU70, oligonucleotides Rg70r3 and Rg70f3 were used as gene-specific primers for 5' and 3' RACE respectively. Two more steps of $5^{\prime}$ RACE using oligos Rg70r4 and Rg70r5 were performed before the full-length cDNA sequence was assembled. Similarly, oligos Rg80r2 and Rg80f2 were used as gene specific primers for $5^{\prime}$ and 3' RACE for KU80 respectively. Another two steps of $5^{\prime}$ RACE were performed using primers Rg80r3 and Rg80r4 to assemble the complete cDNA sequence. All oligonucleotides used are listed in Table 4.

\section{DNA constructs}

All restriction and modification enzymes used were from New England Biolabs Inc. (NEB, Ipswich, MA, USA), unless otherwise stated. Plasmid pEX2 is a pPZP200 derivative routinely used as the binary T-DNA vector backbone [34].

pEX2 was digested with SacI (blunt-ended) and PmeI to remove the hygromycin resistant gene cassette (Pgpd:: $h p t:: \mathrm{T}_{35 S}$ ), and inserted with the 3,618 bp $5^{\prime}$-phosphorylated KU70 DNA fragment amplified from genomic DNA of $R$. toruloides ATCC 10657 using oligos Rg70Lf and Rg70Rr to create pEX2KU70. pDXP795hptR contained a hygromycin selection cassette composed of the endogenous GPD1 promoter from $R$. toruloides (795 bp version), codon-optimized hygromycin phosphotransferase gene (JQ806387, hpt-3) and the terminator of nopaline synthase gene of $A$. tumefaciens ( $\mathrm{P}_{G P D 1}:: h p t-3:: \mathrm{T}_{\text {nos }}$, Additional file $5 \mathrm{~A}$ ), and the selection cassette is flanked at both ends by loxP sites, allowing its deletion by Cre recombinase that can be activated when required (Liu et al., unpublished data). To create $K U 70$ deletion vector pKOKU70, hygromycin selection cassette was digested from pDXP795hptR by BamHI-HindIII and the bluntended fragment was inserted into SmaI-SacI (bluntended) sites of pEX2KU70. Similarly, pKOCAR2 was constructed first by cloning the 2,697 bp $5^{\prime}$-phosphorylated CAR2 fragment into $\mathrm{pEX} 2$, which was amplified using oligos Rt079 and Rt080. Subsequently, the $\mathrm{P}_{G P D 1}:$ : $h p t-3:: \mathrm{T}_{\text {nos }}$ cassette was inserted between SacII-ApaI (blunt-ended) sites, generating pKOCAR2 containing a homologous flanking sequence of approximately $750 \mathrm{bp}$ at both ends. Gene deletion vectors for CAR2 carrying homology sequence of various lengths (50, 100, 250, 500, 750, 1000 and $1500 \mathrm{bp)}$ were likewise constructed using oligonucleotides listed in Table 4 (Additional file 2). For CAR2 complementation, a 3,242 bp fragment amplified by oligos C1500f and Rt080 was 5'-phosphorylated and inserted to HindIII digested and blunt- 
Table 4 Oligonucleotides used

\begin{tabular}{|c|c|}
\hline Name & Sequence $\left(5^{\prime}\right.$ to $\left.3^{\prime}\right)$ \\
\hline Rg70f3 & GTTCATCCCGAGGACCATCAGTC \\
\hline Rg70r3 & GGGATGAGCTCCTTGAGCTTTGC \\
\hline Rg70r4 & TCTTCGTCCGGGTAGATGAAGTAG \\
\hline Rg70r5 & ATCCTTTGGGCTGGCGATGACTTTG \\
\hline Rg80f2 & GTCGCTTGAGCGCATCTTCAACAAG \\
\hline Rg80r2 & ATCACGATGTCCTCGTCCTCGTCGT \\
\hline Rg80r3 & СTCCAACCTGCGGACATGCTTCTT \\
\hline Rg80r4 & GAAGCGAGCCTTGCGCTTGATCTCG \\
\hline Rg70Lf & CTCGTGTCAAGAAAACCAGCAAG \\
\hline Rg70Rr & ACCTCAGCAACCAACCTACCAGT \\
\hline C50f & TCCTCCTACCGACGCTCTACC \\
\hline C50r & CTCTGTGCAGCCATCTCGTTC \\
\hline C10of & TTСATGCCTCAACCCACATC \\
\hline C100r & CGTCCCCAATGCTGAGATAAA \\
\hline C250f & CAAAGTTGGAGGAGGGAGGAG \\
\hline C250r & GTTGAGAAACCAGCGAGACCA \\
\hline C500f & CCTGGGACTCGTACCTCATCC \\
\hline C500r & GCGACCGCGACTGACTAGTTा \\
\hline Rt079 & GGACTGGACTACTGGCTCGTGT \\
\hline
\end{tabular}

Rt080 TCAAGAGCTACCAGGAGAGCAAC

C1000f GGGGCTGTCTGTGACTTGCTAT

C1000r TCGAGCTCAAGCTGAAGAAGAAG

C1500f CTCTCGCACGGGGAGGTAGT

C1500r

TGCTTGCCAAGAAGCCTACAATA

STE2OLf GCTCGTGTTGCGGGAACAGT

STE20Rr GGGCACGCCTTCTTCAAGAAC

Rt033 GTCAAGCCGCCCAGGCTGTC

Rt034 GGATCCGCCAAGTCGCGCAG

Rg70r2 GTCCTTCTTCACCACGCGTTTCTT

Rt096 CGAATCCAACATCGACATGATTT

Rt097 TATTGACGAGCTGAAGAGCCTGT
Specificity

Sense primer for KU70 $3^{\prime}$

RACE Specific primer for

$\triangle$ ku70 fungi colony PCR

Antisense primer 1 for KU70 5' RACE

Antisense primer 2 for KU70 5' RACE

Antisense primer 3 for KU70 5' RACE

Sense primer for KU80 3' RACE

Antisense primer 1 for KU80 5' RACE

Antisense primer 2 for KU80 5' RACE

Antisense primer 3 for KU80 5' RACE

KU70 gene deletion region

CAR2 deletion region

(50 bp homology length)

CAR2 deletion region (100 bp homology length)

CAR2 deletion region (250 bp homology length)

CAR2 deletion region (500 bp homology length)

CAR2 deletion region (750 bp homology length)

CAR2 deletion region (750 bp homology length) CAR2 complementation region

CAR2 deletion region (1000 bp homology length)

CAR2 deletion region

(1500 bp homology length) CAR2 complementation region

CAR2 deletion region (1500 bp homology length)

STE20 deletion region

URA3 deletion region

Specific primer for $\triangle \mathrm{ku} 70$ fungi colony PCR

Specific primer for $\triangle \mathrm{car} 2$ fungi colony PCR
Table 4 Oligonucleotides used (Continued)

\begin{tabular}{llc}
\hline Rt006 & TCTCCCTCGCCCTCTGCT & $\begin{array}{c}\text { Specific primer for GPD1 } \\
\text { (reference gene) in } \\
\text { multiplex PCR } \\
\text { Rt007 }\end{array}$ \\
AGCCATGCCGGTGAGCTTG & $\begin{array}{c}\text { KU70 probe for } \\
\text { Rt100 }\end{array}$ & GACCCGACAGACG blot \\
Rt101 & AGGTCCTTGAGAAAGCGGATG & CAR2 probe for \\
Rt083 & CTCACCCTCGTGTTGCTCGTA & Southern blot \\
Rt084 & CCTCTCCTCCCTCCTCCAACT & \\
\hline
\end{tabular}

ended pDXP795hptR to generate the complementation plasmid (Additional file 5B).

Using the same strategy for gene deletion vectors, the deletion region of STE2O and URA3 were amplified using oligos STE20Lf/STE20Rr (2,196 bp) and Rt33/Rt34 (2,784 bp), cloned into pEX2 and digested using BspHI/ $\mathrm{NcoI}$ and StuI/MfeI (blunt-ended) to create pKOSTE20 and PKOURA3, respectively.

\section{Transformation and identification of transformants}

ATMT and fungal colony PCR were both performed as described previously [6]. For further identification of gene deletion mutants, multiplex PCR [35] using genomic DNA as the template was performed to prevent false negative results. Two sets of primer pairs, one specific to the deletion target (Rg70f3/Rg70r2 and Rt096/Rt097 for $K U 70$ and CAR2 gene, respectively) and the other to the reference gene GPD1 (Rt006 and Rt007) were added to the reactions.

\section{Isolation of genomic DNA, RNA and Southern blot analysis}

Cell cultures at exponential stage were collected and genomic DNA was extracted using MasterPure ${ }^{\text {TM }}$ Yeast DNA purification kit (Epicentre, Madison, WI, USA), while RNA was extracted as described previously [6]. The concentrations of extracted DNA or RNA samples were determined with NanoDrop ND-1000 Spectrophotometer (Thermo Scientific, Wilmington, DE, USA) and their integrity were checked by agarose gel electrophoresis.

For Southern blot analysis, $10 \mu \mathrm{g}$ of genomic DNA was digested with $P v u \mathrm{I}$ at $37^{\circ} \mathrm{C}$ for about $24 \mathrm{hrs}$ and resolved by electrophoresis in a $0.8 \%$ agarose gel. Southern hybridization and detection procedures were performed using DIG (digoxigenin)-High Prime DNA Labeling and Detection kit in accordance with the manufacturer's instructions (DIG Application Manual for Filter Hybridization, Roche Diagnostics, Indiana, IA, USA). The probes were amplified by PCR labeling using DIG DNA labeling mix, with primers Rt100 and Rt101 used to amplify a fragment targeting the $5^{\prime}$ flanking sequence of KU70, and Rt083 and Rt084 specific to the $5^{\prime}$ flanking sequence of CAR2. 


\section{Sensitivity to DNA-damaging agents}

MMS and UV radiation were the DNA-damaging agents used to analyze strain sensitivity monitored by spot plate assay. Cell cultures in YPD broth were adjusted to one $\mathrm{OD}_{600}$ unit and 10-fold serial diluted, from which the diluted samples were spotted on YPD agar plates supplemented with MMS (Sigma, MO, USA) ranging from $0.001-0.1 \%$. Exposure to UV radiation was done by placing the plates in a UV Crosslinker (Spectrolinker ${ }^{\mathrm{rm}} \mathrm{XL}-1000$, Spectronics Corporation, NY, USA) at a dose ranging from 100 to $600 \mathrm{~J} / \mathrm{m}^{2}$ after the samples were spotted.

\section{Photomicroscopy}

Freshly cultured cells were analyzed using a Nikon Eclipse 80i microscope equipped with CFI Plan Apochromat objectives (Nikon, Melville, NY, USA). Images were acquired with a Nikon DS camera interfaced with NIS-Element F 3.0 software.

\section{GenBank accession numbers}

The annotated KU70 and KU80 sequences from R. toruloides ATCC 204091 have been deposited in GenBank under the accession number of KF850470 and KF850471, respectively.

\section{Additional files}

Additional file 1: Colony colors of $\triangle$ car2e after being transformed with a wild type copy of the $R$. toruloides CAR2 genomic DNA fragment. $\triangle$ car2e is a hygromycin sensitive derivative of a CAR2 targeted deletion mutant made by activating the Cre recombinase gene stably integrated into the genome.

Additional file 2: Schematic diagram of CAR2 deletion constructs with varied homology length sequence ranging from 50 to $1500 \mathrm{bp}$ used to compare the homologous recombination frequencies between WT and KU70-deficient strain. Restriction enzyme digest sites used for cloning and Southern blot analysis are indicated. The components in the diagram are not drawn to scale.

Additional file 3: Comparisons of WT and $\Delta$ ku70 strains. (A) Cell morphology; (B) growth rate; (C) sugar consumption rates; (D) fatty acid profiles.

Additional file 4: Comparison of gene deletion frequency between different WT and KU70-deficient fungal stains.

Additional file 5: (A) Schematic illustration of T-DNA region of pDXP795hptR. Unique restriction enzyme digest sites used are shown. (B) Schematic illustration of CAR2 complementation plasmid within T-DNA region.

\section{Competing interests}

The authors declare that they have no competing interests.

\section{Authors' contributions}

CMJK, $L$, and $Y L$ designed the experiments and prepared the manuscript. CMJK, YL, HSM and MD performed the experiments. CMJK, YL and $L$ analyzed the data. All authors read and approved the final manuscript.

\section{Acknowledgements}

This material is based on research supported in part by the Singapore National Research Foundation under CRP Award No. NRF-CRP8-2011-02, the Singapore Economic Development Board and Temasek Trust. We thank
Professor Mark Featherstone, Nanyang Technological University, Singapore, for the kind discussions of the work.

Received: 29 November 2013 Accepted: 21 February 2014 Published: 27 February 2014

\section{References}

1. Sampaio JP, Gadanho M, Bauer R, Weiß M: Taxonomic studies in the Microbotryomycetidae: Leucosporidium golubevii sp. nov., Leucosporidiella gen. nov. and the new orders Leucosporidiales and Sporidiobolales. Mycol Prog 2003, 2:53-68

2. Li Y, Zhao ZK, Bai F: High-density cultivation of oleaginous yeast Rhodosporidium toruloides Y4 in fed-batch culture. Enzyme Microb Tech 2007, 41:312-317.

3. Zhu Z, Zhang $S$, Liu H, Shen $H$, Lin X, Yang F, Zhou YJ, Jin G, Ye M, Zou H, Zhao ZK: A multi-omic map of the lipid-producing yeast Rhodosporidium toruloides. Nat Commun 2012, 3:1112

4. Ratledge C, Wynn JP: The biochemistry and molecular biology of lipid accumulation in oleaginous microorganisms. Adv Appl Microbiol 2002, 51:1-44.

5. Ageitos J, Vallejo J, Veiga-Crespo P, Villa T: Oily yeasts as oleaginous cell factories. Appl Microbiol Biotechnol 2011, 90:1219-1227.

6. Liu Y, Koh CMJ, Sun L, Hlaing M, Du M, Peng N, Ji L: Characterization of glyceraldehyde-3-phosphate dehydrogenase gene RtGPD1 and development of genetic transformation method by dominant selection in oleaginous yeast Rhodosporidium toruloides. Appl Microbiol Biotechnol 2012, 97:1-11.

7. Meyer $\mathrm{V}$ : Genetic engineering of filamentous fungi - Progress, obstacles and future trends. Biotechnol Adv 2008, 26:177-185.

8. Rothstein R: [19] Targeting, disruption, replacement, and allele rescue: Integrative DNA transformation in yeast. In Methods in enzymology. Volume 194. Edited by Christine G, Gerald RF. Academic Press; 1991:281-301.

9. Keeney JB, Boeke JD: Efficient targeted integration at leu1-32 and ura4-294 in Schizosaccharomyces pombe. Genetics 1994, 136:849-856.

10. Shrivastav M, De Haro LP, Nickoloff JA: Regulation of DNA double-strand break repair pathway choice. Cell Res 2008, 18:134-147.

11. Krappmann S: Gene targeting in filamentous fungi: the benefits of impaired repair. Fungal Biology Reviews 2007, 21:25-29.

12. Kück U, Hoff B: New tools for the genetic manipulation of filamentous fungi. Appl Microbiol Biotechnol 2010, 86:51-62.

13. Weld RJ, Plummer KM, Carpenter MA, Ridgway HJ: Approaches to functional genomics in filamentous fungi. Cell Res 2006, 16:31-44.

14. Walker JR, Corpina RA, Goldberg J: Structure of the Ku heterodimer bound to DNA and its implications for double-strand break repair. Nature 2001, 412:607-614.

15. Lieber MR: The mechanism of double-strand DNA break repair by the nonhomologous DNA end-joining pathway. Annu Rev Biochem 2010, 79:181-211.

16. Daley JM, Palmbos PL, Wu D, Wilson TE: Nonhomologous end joining in yeast. Annu Rev Genet 2005, 39:431-451.

17. Modrek B, Lee C: A genomic view of alternative splicing. Nat Genet 2002, 30:13-19.

18. Ninomiya $Y$, Suzuki K, Ishii C, Inoue H: Highly efficient gene replacements in Neurospora strains deficient for nonhomologous end-joining. Proc Natl Acad Sci U S A 2004, 101:12248-12253.

19. Meyer V, Arentshorst M, El-Ghezal A, Drews A-C, Kooistra R, van den Hondel CAMJJ, Ram AFJ: Highly efficient gene targeting in the Aspergillus niger kusA mutant. J Biotechnol 2007, 128:770-775.

20. Goins CL, Gerik KJ, Lodge JK: Improvements to gene deletion in the fungal pathogen Cryptococcus neoformans: Absence of Ku proteins increases homologous recombination, and co-transformation of independent DNA molecules allows rapid complementation of deletion phenotypes. Fungal Genet Biol 2006, 43:531-544.

21. Kretzschmar A, Otto C, Holz M, Werner S, Hübner L, Barth G: Increased homologous integration frequency in Yarrowia lipolytica strains defective in non-homologous end-joining. Curr Genet 2013:1-10.

22. Dujon B, Sherman D, Fischer G, Durrens P, Casaregola S, Lafontaine I, de Montigny J, Marck C, Neuveglise C, Talla E, Goffard N, Frangeul L, Aigle M, Anthouard V, Babour A, Barbe V, Barnay S, Blanchin S, Beckerich J-M, Beyne E, Bleykasten C, Boisrame A, Boyer J, Cattolico L, Confanioleri F, 
de Daruvar A, Despons L, Fabre E, Fairhead C, Ferny-Dumazet H, et al: Genome evolution in yeasts. Nature 2004, 430:35-44.

23. Wilson D, Madera M, Vogel C, Chothia C, Gough J: The SUPERFAMILY database in 2007: families and functions. Nucleic Acids Res 2007, 35:D308-D313.

24. Coelho MA, Rosa A, Rodrigues N, Fonseca A, Gonçalves P: Identification of mating type genes in the bipolar Basidiomycetous yeast Rhodosporidium toruloides: first insight into the MAT locus structure of the Sporidiobolales. Eukaryot Cell 2008, 7:1053-1061.

25. Abbott EP, laniri G, Castoria R, Idnurm A: Overcoming recalcitrant transformation and gene manipulation in Pucciniomycotina yeasts. Appl Microbiol Biotechnol 2013, 97:283-295.

26. Estrada AF, Brefort T, Mengel C, Díaz-Sánchez V, Alder A, Al-Babili S, Avalos J: Ustilago maydis accumulates $\beta$-carotene at levels determined by a retinal-forming carotenoid oxygenase. Fungal Genet Biol 2009, 46:803-813.

27. Hartl L, Seiboth B: Sequential gene deletions in Hypocrea jecorina using a single blaster cassette. Curr Genet 2005, 48:204-211.

28. Goodarzi AA, Noon AT, Deckbar D, Ziv Y, Shiloh Y, Löbrich M, Jeggo PA: ATM signaling facilitates repair of DNA double-strand breaks associated with heterochromatin. Mol Cell 2008, 31:167-177.

29. Noon AT, Shibata A, Rief N, Lobrich M, Stewart GS, Jeggo PA, Goodarzi AA: 53BP1-dependent robust localized KAP-1 phosphorylation is essential for heterochromatic DNA double-strand break repair. Nat Cell Biol 2010, 12:177-184.

30. Hoff B, Kamerewerd J, Sigl C, Zadra I, Kück U: Homologous recombination in the antibiotic producer Penicillium chrysogenum: strain $\triangle \mathrm{Pcku} 70$ shows up-regulation of genes from the HOG pathway. App/ Microbiol Biotechnol 2010, 85:1081-1094.

31. Aparicio OM, Billington BL, Gottschling DE: Modifiers of position effect are shared between telomeric and silent mating-type loci in S. cerevisiae. Cell 1991, 66:1279-1287.

32. Haber JE: Mating-type gene switching in Saccharomyces cerevisiae. Annu Rev Genet 1998, 32:561-599.

33. Lazo GR, Stein PA, Ludwig RA: A DNA transformation-competent Arabidopsis genomic library in Agrobacterium. Nat Biotechnol 1991, 9:963-967.

34. Liu Y, Koh CMJ, Sun L, Ji L: Tartronate semialdehyde reductase defines a novel rate-limiting step in assimilation and bioconversion of glycerol in Ustilago maydis. PLoS One 2011, 6:e16438.

35. Markoulatos $\mathrm{P}$, Siafakas N, Moncany M: Multiplex polymerase chain reaction: A practical approach. J Clin Lab Anal 2002, 16:47-51.

doi:10.1186/1471-2180-14-50

Cite this article as: Koh et al: Molecular characterization of KU70 and KU80 homologues and exploitation of a KU70-deficient mutant for improving gene deletion frequency in Rhodosporidium toruloides. BMC Microbiology 2014 14:50.

\section{Submit your next manuscript to BioMed Central and take full advantage of:}

- Convenient online submission

- Thorough peer review

- No space constraints or color figure charges

- Immediate publication on acceptance

- Inclusion in PubMed, CAS, Scopus and Google Scholar

- Research which is freely available for redistribution 